\section{Stephen Stahl}

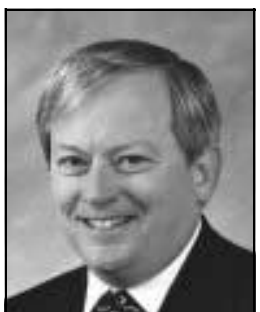

tephen Stahl is adjunct Professor of psychiatry at the University of California, San Diego and Chairman of the Neuroscience

Education Institute. He studied at Northwestern University, Chicago and the University of Chicago and trained at the University of California, San Francisco and Stanford University. His primary research interest is in psychopharmacology.

If you were not a psychiatrist, what would you do?

I would be a novelist, writing mystery novels with mental health-related characters and plots. (I may someday yet reach this goal in retirement.)

\section{Do you feel stigmatised by your profession?}

At the beginning of my career, I was threatened so much by the stigma of psychiatry that I actually trained in neurology first in order to avoid being called a psychiatrist, and thus have the privilege of becoming a 'real doctor'. I eventually decided to train in psychiatry and now, I actually feel no stigma about being a psychiatrist.

\section{What are your interests outside of} work?

I ride, jump and breed warmblood showhorses with my wife on a small horse farm outside of San Diego.

\section{What job gave you the most useful} training experience?

My training at Stanford Medical Center was a key experience in learning to do clinical research and in treating real-world patients with psychopharmacological agents.

What research publication has had the greatest influence on your work?

The body of work by Solomon Snyder on mechanism of action of psychotropic drugs has most influenced my conceptualisation of how the brain works and how to explain it in my textbooks and courses.

\section{What is the most promising} opportunity facing the profession?

Currently, it is the new therapeutic opportunities that are arising from the process of deconstructing psychiatric syndromes into symptoms that reside in circuits regulated by neurotransmitters that can be treated with an ever-increasing cascade of new drugs. This should eventually allow clarification of the genetic control of these pathological symptoms. It might even evolve into disease modifying rather than just symptomatic treatments in psychiatry.

What single change would substantially improve quality of care? More rational combinations of drugs and more rational combinations of drugs with psychotherapy. This is still done à la carte, with a wide range of idiosyncrasies in the field, some of them quite irrational.

\section{What conflict of interest do you} encounter most of ten?

Professional organisations such as the American Psychiatric Association (APA) on the one hand complaining about excessive influence of the pharmaceutical industry, while on the other hand, soliciting and charging exorbitant fees to this same industry without which the organisations would collapse.

What are the main ethical problems that psychiatrists will face in the future?

Providing patients with less than ideal therapeutics because of payer restrictions that are aimed at saving money rather than providing best practice outcomes.

\section{How would you improve clinical} psychiatric training?

More exposure to how highly-effective clinicians solve problems in clinical practice. Too much psychiatric supervision occurs away from patients, with trainees talking about patients they see rather than trainees seeing a master in action.

What single change to mental health legislation would you like to see? Parity is a no brainer. Surgical patients fly first class, medical patients fly coach and psychiatric patients are too often cargo.
How should the role of the American Psychiatric Association change? The APA in particular is an organisation way, way out of touch with its members, and is seen by many l encounter in the field to exist largely for those who run the organisation and not for those in the profession. For this reason, its membership is flat to declining, and young psychiatrists in particular often see little use in joining an extremely expensive organisation and it is hard for me to disagree. Until the organisation is actually member-driven rather than seen by its members to give mere lip service to members in order to survive for itself, the practitioners I meet in lectures and courses have told me that they feel it will remain basically politically oriented and intellectually corrupt. I am an APA distinguished fellow and it is actually an embarrassment to me to have the APA so out of favour with its members.

What is the future for psychotherapy in psychiatry training and practice? Psychotherapy will continue to have a central role in training, but will continue to have a peripheral role in third party reimbursement in practice. A well-rounded psychiatrist must have psychotherapy skills that can be utilised even in intake interviewing and will be able to provide some services directly to self-paying patients and supervise effectively the therapy of lowercost providers; the reality in the world today.

What single area of psychiatric research should be given priority? Brain imaging and linking circuits to neurotransmitters, and symptoms and drugs should hold the most promise for understanding psychiatric disorders and enhance the chances of developing disease modifying or preventative therapeutics in the future.

What single area of psychiatric practice is most in need of development?

Integration of psychiatry into mainstream medicine. The field remains too peripheral to other specialities and it would be mutually beneficial if psychiatry does not continue to be the stepchild of medical specialties.

Dominic Fannon 